\title{
High Quality Integrated Data Reconstruction for Medical Applications
}

\author{
A.K.M Fazlul Haque \\ Department of Computer Science \\ and Engineering, \\ Jahangirnagar University, \\ Dhaka, Bangladesh. \\ Email: akm_haque@yahoo.com
}

\author{
Md. Hanif Ali \\ Department of Computer Science \\ and Engineering \\ Jahangirnagar University, \\ Dhaka, Bangladesh. \\ Email: hanif_ju03@yahoo.com
}

\author{
M Adnan Kiber \\ Department of Applied Physics, \\ Electronics and Communication \\ Engineering, University of Dhaka, \\ Bangladesh. \\ Email:ma_kiber@yahoo.com
}

\begin{abstract}
In this paper, the implementation of a high quality integrated data reconstruction model and algorithm has been proposed, especially for medical applications. Patients' Information acquired at the sending end and reconstructed at the receiving end by using a technique that would be high quality for the signal reconstruction process. A method is proposed in which the reconstruction of data like ECG, audio and other patients' vital parameters that are acquired in the time-domain and operated in the frequency-domain. Further the data will be reconstructed in the time-domain from the frequency domain where high quality data is required. In this particular case, high quality ensures the distortion less and noiseless recovered baseband signal. This would usually require the application of Fast Fourier Transform (FFT) and Inverse Fast Fourier Transform (IFFT) return the data to the spatial domain. The simulation is performed using Matlab. The Composite baseband signal has been generated by developing a program as well as by acquiring to the workspace. The feature of the method is that it can achieve high-quality integrated data reconstruction and can be associated easily with spatial domain.
\end{abstract}

Keywords: FFT, IFFT, ECG, Baseband, Reconstruction, Noise, FDA tool.

\section{INTRODUCTION}

High quality integrated data reconstruction model and algorithm are used, within an electronic system, to extract the desired time-domain signal from the frequency-domain signal acquired from the human body (in offline), especially Electrocardiogram (ECG), audio and other vital parameters [1]. Each stage automatically generates a template of a source from the candidate events in the initialization period, and thereafter performs classification of the remaining candidate events based on a template matching technique. Matlab simulation results on offline demonstrate the effectiveness of the proposed method.

In recent literature [2, 3, 4], the perception of conveying vital information reconstruction used by medical practitioners has had some concentration. $\mathrm{Li}$, Mueller and Ernst [2] emphasized on the methods for efficient, high quality volume resampling in the frequency domain. It was described in the use of frequency-domain filters for the accurate resampling of images and volumes at arbitrary magnification factor. It was also investigated the Freq approach in relation to higher-quality filters.

Chazan, Hoory, Cohen, and Zibulski [3] illustrated speech reconstruction from Mel frequency cepstral coefficients and pitch frequency. They presented a novel low complexity, frequency domain algorithm for reconstruction of speech from the Mel frequency cepstral coefficient. The construction technique was based on the sinusoidal speech representation.

Kong [4] focused on GPS modeling in Frequency Domain. The author presented a frequency domain modeling approach to model GPS errors and increase GPS positioning shaping filter. The application of approach was mainly used for vehicle navigation system.

In this paper, most of the information contained in the baseband signal is found below $100 \mathrm{~Hz}$. High frequencies random noise may corrupt the reconstructed time domain baseband signal. To remedy the situation, Filter Design Tool (FDA) has been used to eliminate the high frequency component. There is no distortion appearing in that particular spatial domain instead of attenuation amplified by the gain of the signal.

Bioelectrical signals are typically very small in amplitude $(\mathrm{mV})$ and an amplifier is required to accurately depending on the hardware and software used, the biological amplifier serves to amplify the signal. It is also known that the frequency of heart signals is very low, approximately 5 to $10 \mathrm{H}_{\mathrm{z}}[5,6]$.

\section{MATERIALS AND METHODS}

The reconstruction process would be in order of data collection, Discrete Fourier transform (DFT), Fast Fourier Transform (FFT), Inverse Fourier Transform (IFFT), and finally, noise cancellation. These topics are discussed below:

The discrete Fourier transform, or DFT, is the primary tool of digital signal processing. The foundation of the Signal Processing Toolbox is the fast Fourier transform 
(FFT), a method for computing the DFT with reduced execution time [7, 8]. Many of the toolbox functions (including z-domain frequency response, spectrum and cepstrum analysis, and some filter design and implementation functions) incorporate the FFT. MATLAB provides the functions FFT and IFFT to compute the discrete Fourier transform and it's inverse, respectively. For the input sequence $\mathrm{x}$ and its transformed version $\mathrm{X}$ (the discrete-time Fourier transform at equally spaced frequencies around the unit circle), the two functions implement the relationships.

$$
\begin{aligned}
& X(k+1)=\sum_{n=0}^{N-1} x(n+1) W_{N}^{k n} \\
& x(n+1)=\frac{1}{N} \sum_{k=0}^{N-1} X(k+1) W_{N}^{-k n}
\end{aligned}
$$

In these equations, the series subscripts begin with 1 instead of 0 because of the MATLAB vector indexing scheme, and

$$
W_{N}=e^{-j\left(\frac{2 \pi}{N}\right)}
$$

Working with the Fourier transform on a computer usually involves a form of the transform known as the discrete Fourier transform (DFT). There are two principal reasons for using this form: The input and output of the DFT are both discrete, which makes it convenient for computer manipulations. There is a fast algorithm for computing the DFT known as the fast Fourier transform (FFT).

The DFT is usually defined for a discrete function $\mathrm{f}(\mathrm{m}$, n) that is nonzero only over the finite region $0<=\mathrm{m}<=\mathrm{M}$ and $0<=\mathrm{n}<=\mathrm{N}$. The two-dimensional M-by-N DFT and inverse M-by-N DFT relationships are given by

The values $F(p, q)$ are the DFT coefficients of $f(m, n)$.

$$
\begin{aligned}
& F(p, q)=\sum_{m=0} \sum_{n=0}^{M-1} f(m, n) e^{-j(2 \pi / M) p m} e^{-j(2 \pi / N) q n} \quad \begin{array}{ll}
p=0,1, \ldots, M-1 \\
q=0,1, \ldots, N-1
\end{array}
\end{aligned}
$$

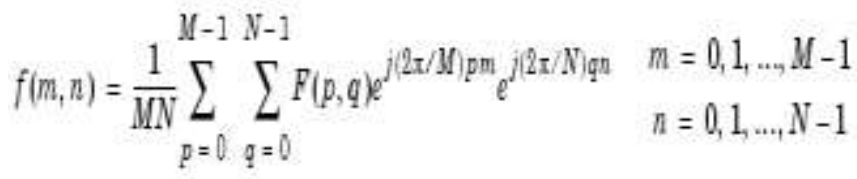

The zero-frequency coefficient, $\mathrm{F}(0,0)$, is often called the "DC component." DC is an electrical engineering term that stands for direct current. (Note that matrix indices in
MATLAB always start at 1 rather than 0 ; therefore, the matrix elements $f(1,1)$ and $F(1,1)$ correspond to the mathematical quantities $f(0,0)$ and $F(0,0)$, respectively.)

The DFT coefficients F (p , q) are samples of the Fourier transform $\mathrm{F}\left(\omega_{1}, \omega_{2}\right)$.

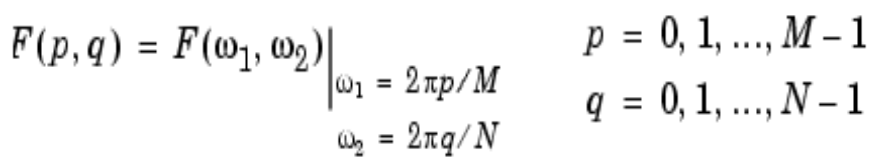

\section{DATA COLLECTION}

Data collection process can be acquired by real time or offline. In real time, data, especially ECG are acquired by data acquisition tools. Other vital parameters like audio, video can be acquired by existing audio-video editing software [9]. If the existing software does not support to acquire the above parameters, it can be considered for developing the software to perform the acquisition properly. The array of electrodes used for the capture of Body Surface Potentials (BSP) comprises a flexible plastic anterior and posterior electrode harness and a portable recording unit. The anterior harness contains 64 electrodes, including 3 proximal bipolar limb leads (Mason-Likar position), and a posterior harness with 16 electrodes. This lead configuration enables recording of 77 unipolar ECG signals with respect to the Wilson central terminal. Recordings are taken over $4198 \mathrm{~ms}$, the sampling rate is $1 \mathrm{kHz}$ and the potentials are recorded using a 12-bit resolution to represent a $5 \mathrm{mV}$ range. The procedure for attaching the harnesses to a patient and taking measurements of electrical potentials lasts about 3-4 minutes [10].

In this regard, data have been taken off-line generated by the software Matlab. It is worth mentioning that different data carries the different information that would be recovered by the method given in the next part of this paper.

\section{PROPOSED METHOD}

The algorithm is based on using a sinusoidal model, where audio signal and ECG are represented by workspace block converted the captured file, especially .wav file into 2dimentional data file. signals are characterized by the frequencies, amplitudes and phases of the sine-wave components. The complete reconstruction scheme is presented in Figure. 1. It is applied in every $10 \mathrm{~ms}$. frame using the simulink block system's rules and regulations.

The algorithm is comprised of the following stages:

1. Sum block - Add or subtract inputs. Specify one of the following:

a) String containing + or - for each input port.

b) Scalar $>=1$. A value $>1$ sums all inputs; 1 sums elements of a single input vector. 
2. FFT - Outputs the complex Fast Fourier Transform (FFT) of real or complex input by computing radix-2 decimation-in-time (DIT) or decimation-in-frequency (DIF), depending on block options. Uses half-length and doublesignal algorithm for real inputs where possible. Computes the FFT outputs of the Digital Filter block, the filter function in MATLAB, and the filter function in the Filter Design Toolbox. The sampling frequency, Fs, which specified in the FDA tool's Graphical User Interface (GUI), should be identical to the sampling frequency of the digital filter design block's input block. When the sampling frequencies

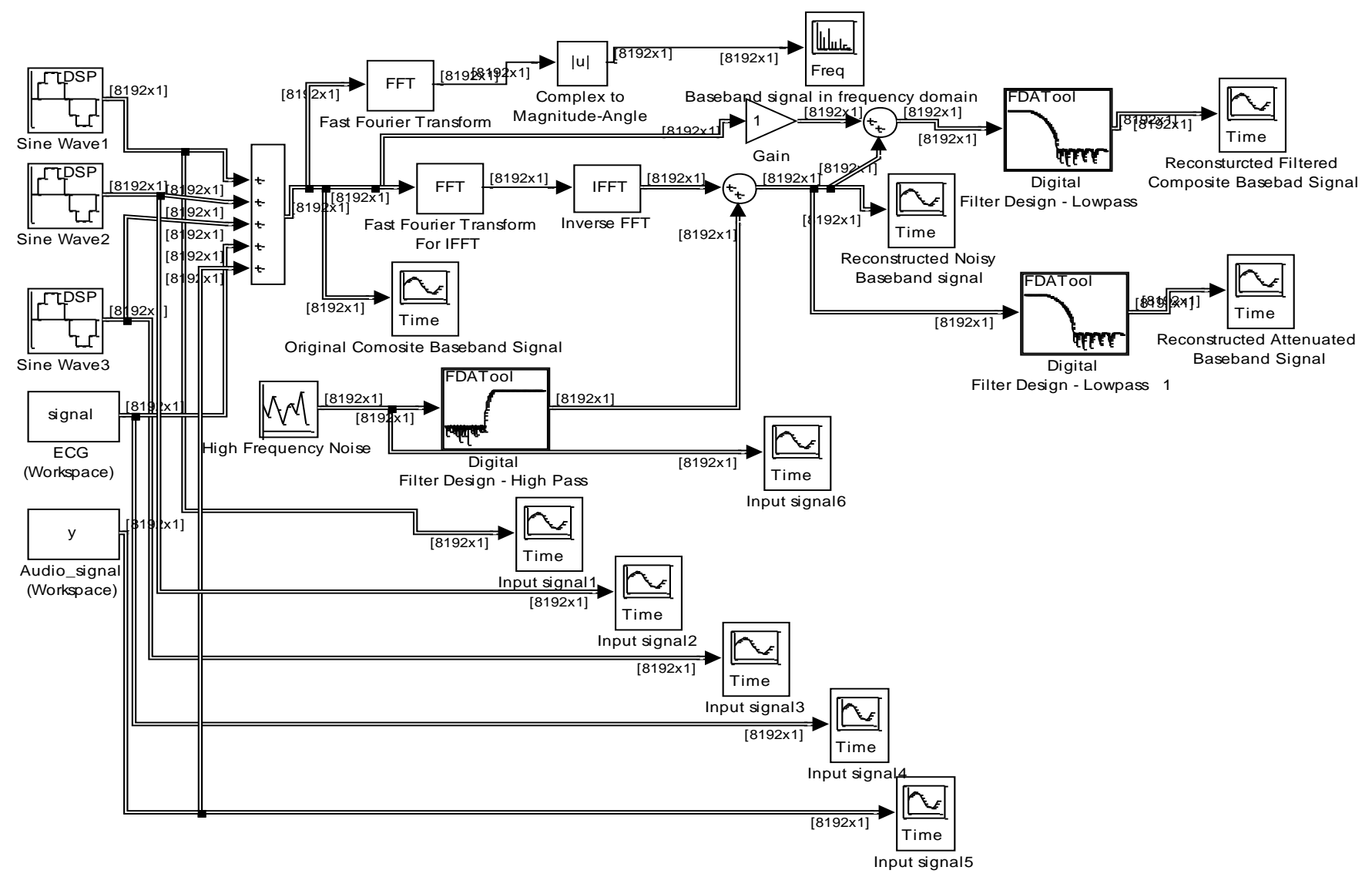

Figure 1. Block Diagram of proposed simulation method

along the vector dimension for sample-based vector inputs, which must have a power-of- 2 length. Computes the FFT along each column for all other inputs, where columns must be a power-of-2 length.

3. IFFT - Outputs the Inverse Fast Fourier Transform (FFT) of real or complex input by computing radix-2 decimation-in-time (DIT) or decimation-in-frequency (DIF), depending on block options.

Outputs are real if we select 'Input is conjugate symmetric' option; otherwise, outputs are complex. Computes the IFFT along the vector dimension for samplebased vector inputs, which must have a power-of- 2 length. Computes the IFFT along each column for all other inputs, where columns must be a power-of- 2 length.

4. FDA Tool - The block applies the specified filter to each channel of a discrete-time input signal, and outputs the result. The outputs of the block numerically match the of these blocks do not match, the digital filter design block returns a warning message and inherits the sampling frequency of the input block.

The block accepts inputs that are sample-based or framebased vectors and matrices. The block filters each input channel independently over time, where

- Each column of a frame-based vector or matrix is an independent channel.

- Each element of a sample-based vector or matrix is an independent channel.

5. Workspace - Simulink allows us to import input signal and initial state data from the MATLAB workspace and export output signal and state data to the MATLAB workspace during simulation. This capability allows us to use standard or custom MATLAB functions to generate a simulated system's input signals and to graph, analyze, or otherwise postprocess the system's outputs STFT reconstruction (Fig. 1) - The frequencies, phases and 
amplitudes are combined to form a sine-wave representation. The final reconstructed STFT is constructed from the sine waves by a convolution procedure.

To use this format, it selects input in the load from workspace pane and selects the Array option from the Format list on the data import/export pane. Selecting this option causes Simulink to evaluate the expression next to the Input check box and use the result as the input to the model. The expression must evaluate to a real (noncomplex) matrix of data type double. The first column of the matrix must be a vector of times in ascending order. The remaining columns specify input values. In particular, each column represents the input for a different Import block signal (in sequential order) and each row is the input value for the corresponding time point. Simulink linearly interpolates or extrapolates input values as necessary if the Interpolate data option is selected for the corresponding import. The total number of columns of the input matrix must equal $n+1$, where $n$ is the total number of signals entering the model's imports.

6. Vector Scope - The vector scope block is a comprehensive display tool similar to a digital oscilloscope. The block can display time-domain, frequency-domain, or user-defined signals. We can use the Vector Scope block to plot consecutive time samples from a frame-based vector, or to plot vectors containing data such as filter coefficients or spectral magnitudes. To compute and plot the periodogram of a signal with a single block, use the Spectrum Scope block. The input to the Vector Scope block can be any realvalued $\mathrm{M}-\mathrm{by}-\mathrm{N}$ matrix, column or row vector, or 1-D (unoriented) vector, where 1-D vectors are treated as column vectors. Regardless of the input frame status, the block treats each column of an $\mathrm{M}-$ by- $\mathrm{N}$ input as an independent channel of data with $\mathrm{M}$ consecutive samples. The block plots each sample of each input channel sequentially across the horizontal axis of the plot conversion to time domain (Fig. 1) - This is the final stage in which the time domain signal frame is computed.

7. Sine wave - The sine wave block may generate a multichannel real or complex sinusoidal signal, with independent amplitude, frequency, and phase in each output channel. A real sinusoidal signal is generated when the output complexity parameter is set to Real, and is defined by an expression of the type

$$
y=A \sin (2 \pi f t+\varphi)
$$

where $\mathrm{A}$ in the amplitude parameter, $\mathrm{f}$ in hertz in the frequency parameter, and in radians in the phase offset parameter. A complex exponential signal is generated when the output complexity parameter is set to complex, and is defined by an expression of the type

$$
y=A e^{j(2 \pi f t+\phi)}=A[\cos (2 \pi f t+\phi)+j \sin (2 \pi f t+\phi)\}
$$

or Gaussian (normal) distribution. Set output repeatability to nonrepeatable (block randomly selects initial seed every time simulation starts), repeatable (block randomly selects initial seed once and uses it every time simulation starts), or specify seed (block uses specified initial seed every time simulation, starts producing repeatable output).

\section{ANALYSIS AND DISCUSSION}

The first stage of the implementation is to pass the simulated integrated baseband signal corrupt by high frequency random noise. The method tries to produce the filtered simulated distortion less and noiseless integrated baseband signal in a relatively short period of time. Increasing the filter order above one slows down the convergence rate but makes the results more precise. The recovered signal closely resembles the original simulated signal minus the noise and hence indicates that the implementation of the algorithm functions correctly and efficiently. The second stage is to recover the original composite baseband attenuated signal using FDA tool. Finally, the original composite banseband signal has been recovered where noise, distortion and attenuation have been eliminated.

High frequency noise is used, as the signal corrupt by noise as well as the reference noise. With this setup, it is observed that the output signal has been attenuated a little bit but not distorted due to the affect of distortion. But the attenuation would be disappeared by amplifying the gain of the signal. All involved steps of the simulation are discussed below.

Step 1: Generation of different sinusoidal signals having amplitude of $1 \mathrm{mV}$, ECG pattern having amplitude of $3.5 \mathrm{mV}$ and audio signal having amplitude of $0-1 \mathrm{mV}$. Figure $2,3,4,5$, and 6 show the generation of input signal. Here ECG signal has been generated in offline and the time domain data imported from the workspace. The data of the audio signal may take from stored file or real time. In this particular case, it has been taken from the stored file and the time domain data also imported from the workspace.

Step 2: The composite baseband signal for the different input signals (Amplitude of $4.5 \mathrm{mV}$ ) has been showed in Fig. 7.

Step 3: Fig. 8 shows the original composite baseband signal in frequency domain.

Step 4: Generation of high frequency random noise signal amplitude of $3.5 \mathrm{mV}$ is shown in Fig. 9.

Step 5: The noisy IFFT signal (recovered baseband signal + noise) is shown in Fig. 10 .

Step 6: Fig. 11 shows the reconstructed filtered attenuated composite baseband signal using FDA tool.

Step 7: Fig. 12 shows the original reconstructed filtered composite baseband signal using FDA tool.

8. Random source - Output a random signal with uniform 


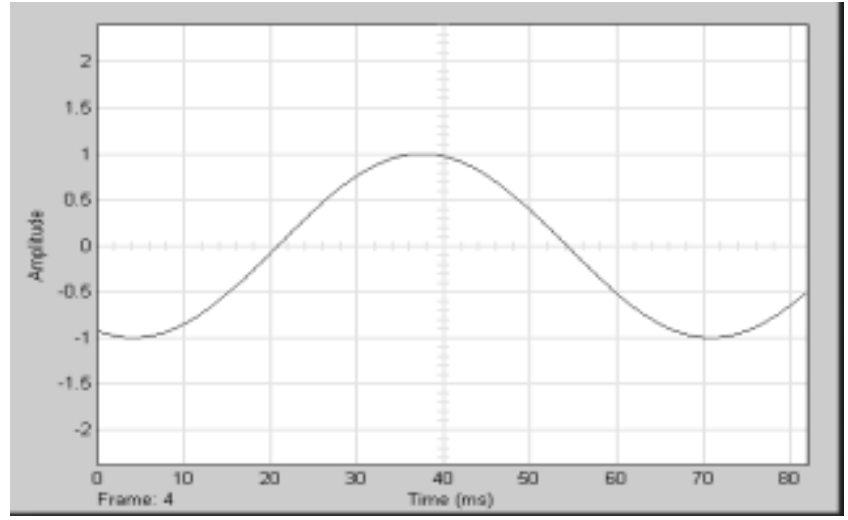

Figure 2. Sinusoidal signal with frequency of $15 \mathrm{~Hz}$

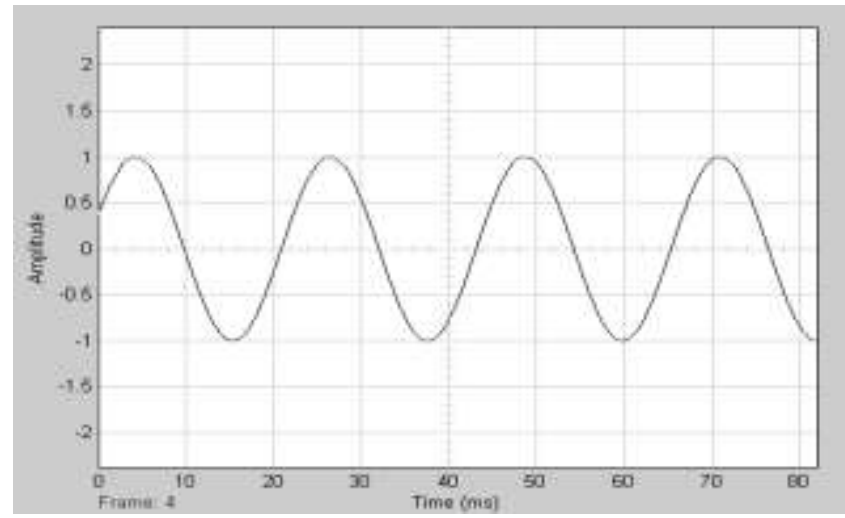

Figure 3. Sinusoidal signal with frequency of $45 \mathrm{~Hz}$

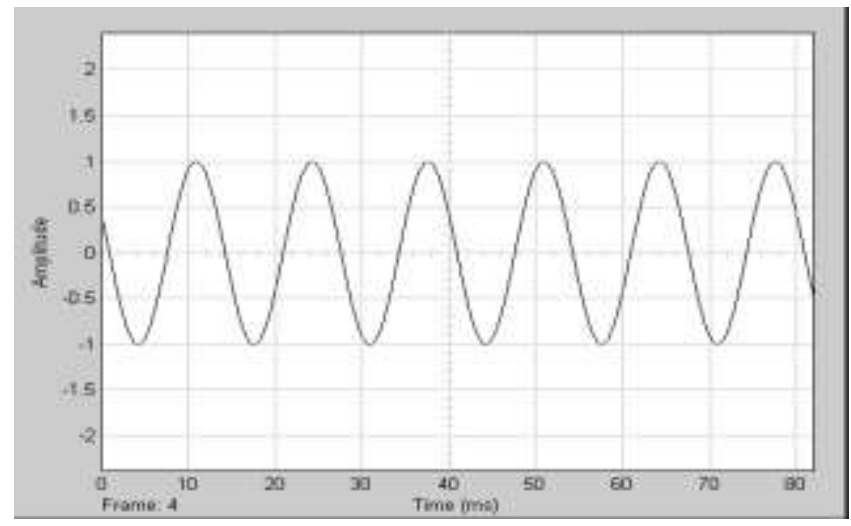

Figure 4. Sinusoidal signal with frequency of $75 \mathrm{~Hz}$

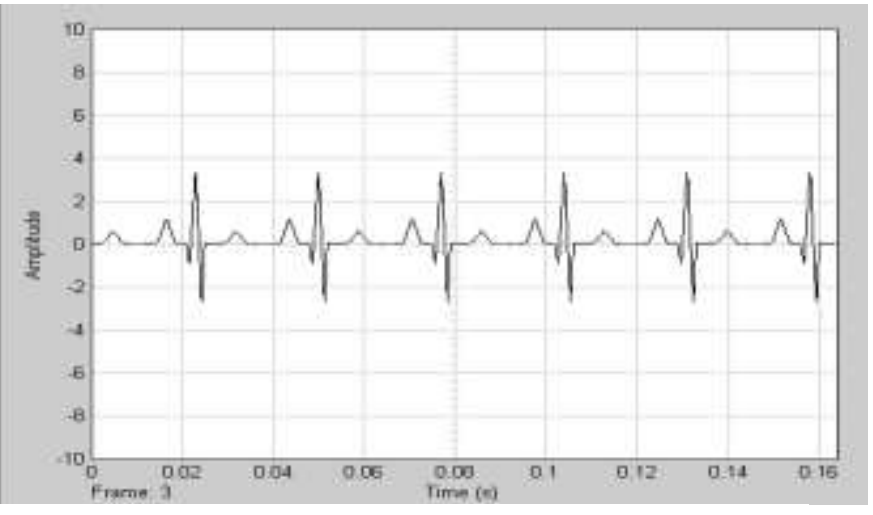

Figure 5. ECG Signal Generated from workspace

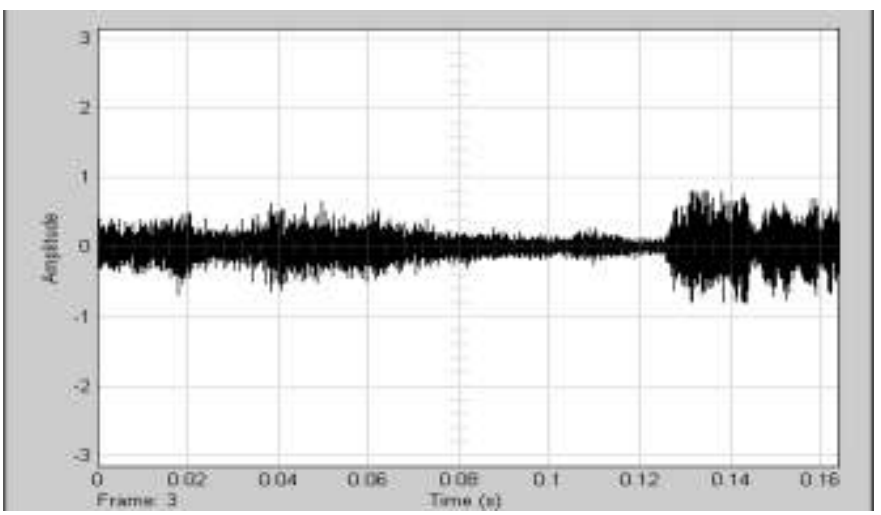

Figure 6. Audio Signal Generated from workspace.

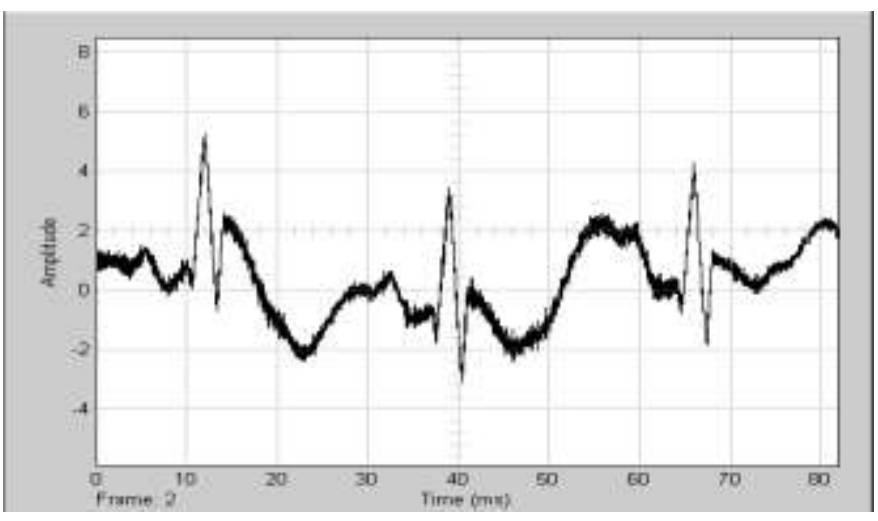

Figure 7. Original Composite Baseband Signal. 


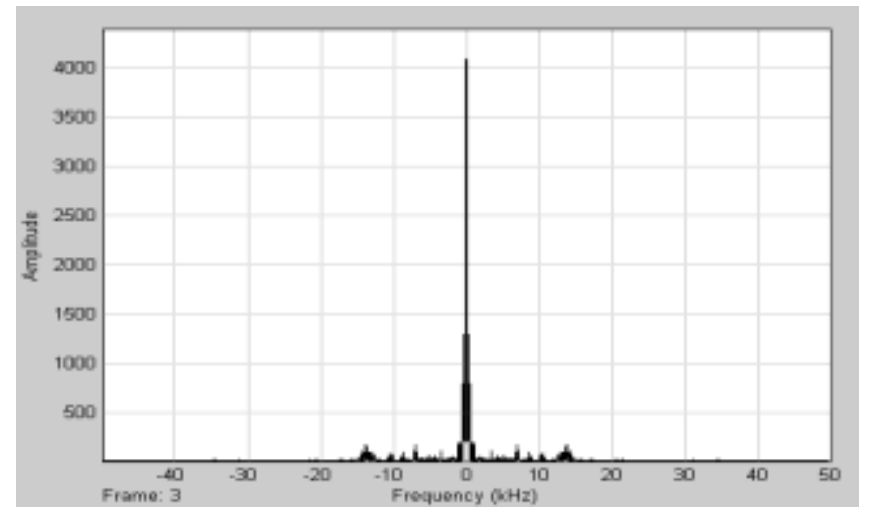

Figure 8. Original Composite Baseband Signal in Frequency Domain.

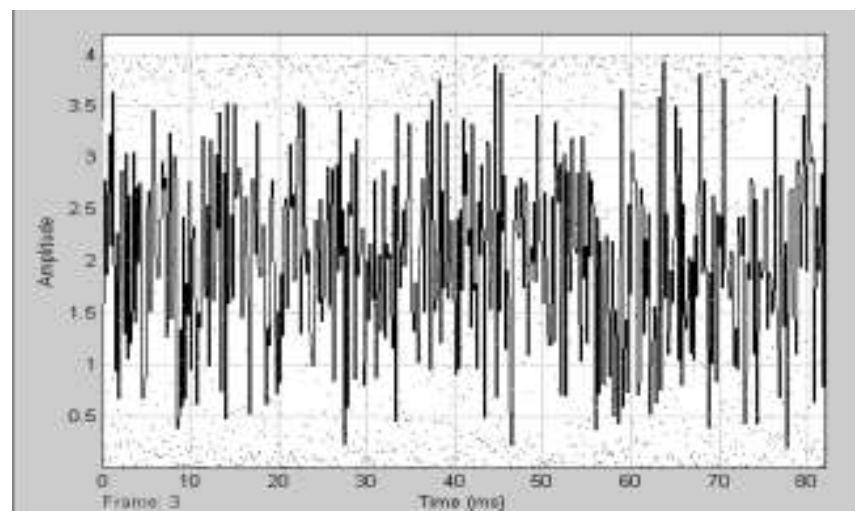

Figure 9. High frequency Random Noise.

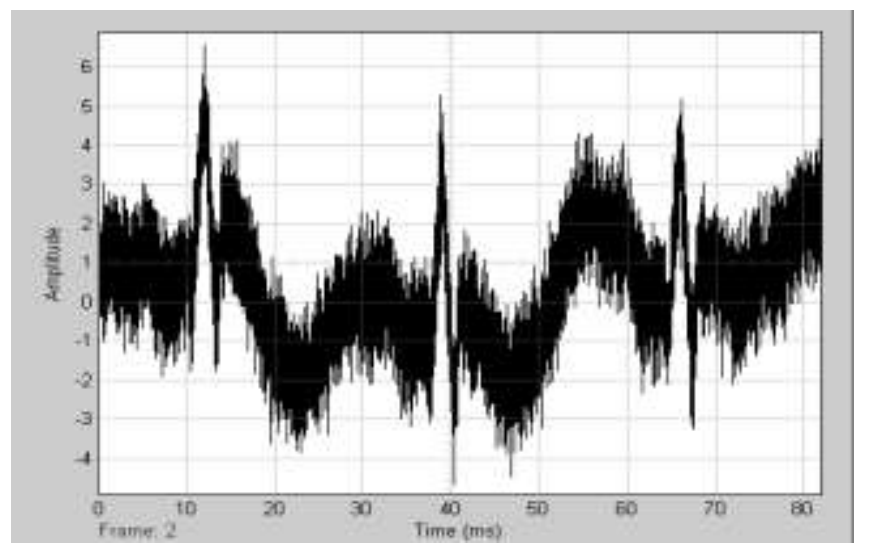

Figure 10. Recovered Noisy Baseband Signal.

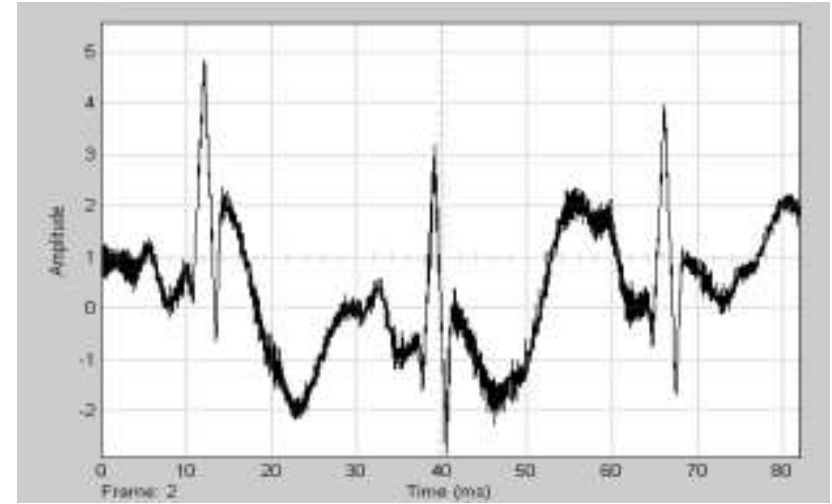

Figure 11. Original Filtered Reconstructed attenuated Signal.

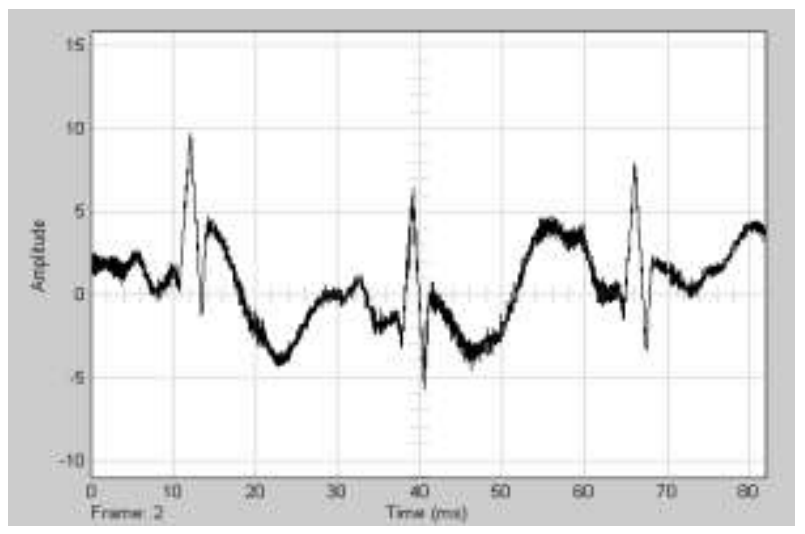

Figure 12. Original Filtered Reconstructed Signal.

\section{CONCLUSION}

In this work, an attempt has been made to employ a method that can be implemented for reconstructing a high quality integrated data, especially for medical applications. Distortions of reconstructed filtered baseband signal may occur due to the affect of frequency offset in integrated data that have been eliminated. Generally, the reconstruction of data like ECG, audio and other patients' vital parameters that are acquired in the time-domain and operated in the frequency-domain. Data acquisition and the simulation are performed using Matlab. The Composite baseband signal, especially ECG, has been generated by developing a program. Filter Design Tool (FDA) has been used to eliminate the high frequency component and the original filtered baseband signal has been obtained. Distortions have been eliminated in that particular spatial domain and attenuation has been eliminated by amplifying the gain of the signal. This approach may be considered for reconstruction in the advanced technology which needs 
further investigation.

\section{REFERENCES}

[1] R. Aston, Principles of biomedical instrumentation and measurement. Columbus: Merrill Pub. Co., 1990.

[2] Aili Li, Klaus Mueller, Thomas Ernst, "Methods for Efficient, High quality Volume Resampling in the Frequency Domain", IEEE Visualization 2004.

[3] Dan Chazan, Ron Hoory, Gilad Cohen and Meir Zibulski, "Speech Reconstruction From Mel Frequency Cepstral Coefficients and Pitch Frequency" Acoustics, Speech, and Signal Processing, Vol 3, IEEE 2000.

[4] Xiaoying Kong, "GPS Modeling in Frequency Domain" presented at $2^{\text {nd }}$ International Conference on Wireless Broadband and Ultra Wideband Communications, IEEE 2007.

[5] Monitoring Patients via Radio Signals. About Wireless Medical Telemetry Service (WMTS).

[6] A.K.M Fazlul Haque, M. Shamim Kaiser, S. K. Aditya, "Background Noise Cancellation Using Adaptive LMS Algorithm" Journal of Bangladesh Electronic Society, Volume 6, Issue 2.

[8] Lianming Sun, Akira Sano, "Identification and Error Analysis in Frequency Domain," Proceedings of the First International Conference on Innovative Computing, Information and Control, IEEE 2006.

[9] Mircea-Florin VAIDA, Ionel STAMATE,'Integrated Medical Image Processing Facilities Using a Dedicated Application" International Conference on Automation, Quality and Testing, Robotics , IEEE 20006.

[10] Sesar Navarro, Colin Turner, Omar Escalona, "A Method for the ECG Inverse Problem in the Frequency Domain" Proceeding of the $18^{\text {th }}$ IEEE Symposium on Computer-Based Medical Systems, IEEE 2005. 